Recepción: 20 / 01 / 2017

Aceptación: 20 / 02 / 2017

Publicación: 15 / 03 / 2017

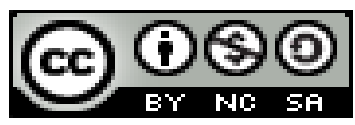

Ciencias Médicas

Articulo de investigación

\title{
Hipotermia y postoperatorio
}

\author{
Hypothermia and postoperative
}

\section{Hipotermia e pós-operatório}

Elio O. Ochoa Gavilánez ${ }^{\text {I }}$ ochoaeliogavilanez@hotmail.com

Carmen S. Olave Matamba II carmencitaolave69@gmail.com

Jenny P. Guzmán Arias III jguzmana@gmail.com

Pedro L. Maldonado Álava IV pedro.maldonadoa@ug.edu.ec

Correspondencia: ochoaeliogavilanez@hotmail.com

\footnotetext{
I. Doctor en Medicina y Cirugía; Hospital General Dr. Liborio Panchana Sotomayor; Santa Elena, Ecuador. Doctor en Medicina y Cirugía; Hospital General Dr. Liborio Panchana Sotomayor; Santa Elena, Ecuador. Médica; Hospital General Dr. Liborio Panchana Sotomayor; Santa Elena, Ecuador.

Magister en Salud Pública; Diplomado en Docencia Superior; Doctor en Medicina y Cirugía; Universidad de Guayaquil, Guayaquil, Ecuador.
} 
Elio O. Ochoa-Gavilánez; Carmen S. Olave-Matamba; Jenny P. Guzmán-Arias; Pedro L. Maldonado-Álava

\section{Resumen}

La anestesia y la cirugía causan un significativo impacto sobre el balance térmico corporal a causa de la alteración de los mecanismos de termorregulación normales y por la pérdida de calor causada durante la cirugía (exposición a un ambiente con bajas temperaturas en la sala de operaciones, soluciones frías de lavado intraoperatorias y de infusiones intravenosa). Numerosos estudios demostraron que la hipotermia perioperatoria aumenta la incidencia de efectos adversos para el paciente: mayor sangrado, deterioro de la calidad de recuperación postoperatoria, mayores tiempos de permanencia en unidad de recuperación postanestésica. También se demostró una mayor incidencia de complicaciones a largo plazo: infección de las heridas quirúrgicas, hospitalización prolongada, isquemia miocárdica, arritmias cardíacas. Este aumento de las complicaciones tiene consecuencias económicas para el sistema de salud y también, posiblemente, sociales al retardar el reintegro de los pacientes a su medio familiar y laboral. El aumento de la temperatura del ambiente, calentamiento de fluidos intravenosos y el calentamiento cutáneo activo son medidas necesarias durante el intraoperatorio para evitar la hipotermia. En esta revisión no sistemática el objetivo es exponer las características y eficacia de los métodos de monitorización y de calentamiento intraoperatorio, resumir los mecanismos.

Palabras clave: Hipotermia; anestesia; termorregulación; medidas de calentamiento. 


\begin{abstract}
Anesthesia and surgery lead to a significant impact in body temperature balance. The mechanisms of this disturbance are the dysfunction of the normal thermoregulatory systems as well as heat loss during surgery (low operating room temperature, cold intravenous or surgical fluid infusions). Several studies have demonstrated that perioperative hypothermia increases adverse outcomes: major bleeding, impairment of quality of recovery after anesthesia, increases length of staying in postoperative recovery room. It has also been demonstrated that hypothermia influences long term outcomes in surgical patients by increasing rate of infections, length of hospitalization, myocardial ischemia and cardiac arrhythmias. These consequences might have important economical consequences for the health system and possibly social implications by delaying patient reinsertion in social life and jobs. Optimizing room temperature, heating intravenous fluids and active skin warming are necessary in the intraoperative setting to minimize hypothermia incidence. In this nonsystematic review we aim to describe temperature monitoring, physiological mechanisms and intraoperative warming strategies to minimize hypothermia during surger.
\end{abstract}

Key words: Hypothermia; anesthesia; thermoregulation; outcomes; warming systems. 
Elio O. Ochoa-Gavilánez; Carmen S. Olave-Matamba; Jenny P. Guzmán-Arias; Pedro L. Maldonado-Álava

\section{Resumo}

A anestesia e a cirurgia levam a um impacto significativo no equilíbrio da temperatura corporal. Os mecanismos desta perturbação são a disfunção dos sistemas termorreguladores normais, bem como a perda de calor durante a cirurgia (baixa temperatura da sala de operação, infusão de fluido intravenoso ou cirúrgico). Vários estudos demonstraram que a hipotermia perioperatória aumenta os resultados adversos: hemorragia maior, comprometimento da qualidade da recuperação após anestesia, aumenta o tempo de permanência na sala de recuperação pós-operatória. Também foi demonstrado que a hipotermia influencia os resultados a longo prazo em pacientes cirúrgicos pelo aumento da taxa de infecções, duração da hospitalização, isquemia miocárdica e arritmias cardíacas. Essas consequiências podem ter importantes conseqüências econômicas para o sistema de saúde e, possivelmente, implicações sociais ao atrasar a reinserção do paciente na vida social e no emprego. A otimização da temperatura ambiente, o aquecimento de líquidos intravenosos e o aquecimento ativo da pele são necessários na configuração intra-operatória para minimizar a incidência de hipotermia. Nesta revisão não-sistemática, procuramos descrever monitoramento de temperatura, mecanismos fisiológicos e estratégias de aquecimento intra-operatório para minimizar a hipotermia durante o cirurgião.

Palavras chave: Hipotermia; anestesia; Termorregulação; resultados; Sistemas de aquecimento. 


\section{Introducción.}

La temperatura corporal es una constante más de los clásicos signos vitales, hablamos de hipotermia cuando la temperatura corporal es menor a 36 grados centígrados, la temperatura comúnmente es subvalorada por la mayoría de médicos en el quirófano, pero en el ámbito anestésico debemos estar atentos por las repercusiones clínicas que puede conllevar tales como : aumento de la incidencia de infección de la herida operatoria, hospitalización prolongada, deterioro de la coagulación y de la inmunidad, isquemia miocárdica, arritmias cardíacas, malestar extremo del paciente , tiempos en la sala de recuperación postanestésica prolongados. $(1,2,3)$

La falta en la monitorización de esta constante vital es la causa de complicaciones desde leves a severas, hay que recordar que hay constantes que hacen variar la temperatura en personas normales como son el sexo, la edad, la hora del día.

Los lugares para medir la temperatura son: temperatura central (cabeza troco y órganos) membrana timpánica (carótida), la nasofaringe, arteria pulmonar (swanganz) y el esófago distal (aorta) estos son invasivos. Sitios que valoran mejor el compartimiento periférico: Boca, axila, recto, vejiga urinaria. Poseen exactitud razonable excepto en situaciones térmicas extremas. (4)

Las fuentes producción de calor dependen de factores externos o indirectos o directos, estos últimos corresponden con la actividad muscular y las reacciones bioquímicas de los procesos metabólicos, mientras que la fuente externa o indirecta está dada por la ganancia del calor del ambiente.

Para que dichos factores mantengan una temperatura constante existe un sistema de retroalimentación positiva y negativa en el sistema nervioso central. 
Elio O. Ochoa-Gavilánez; Carmen S. Olave-Matamba; Jenny P. Guzmán-Arias; Pedro L. Maldonado-Álava

El hipotálamo anterior actúa como el principal regulador de la temperatura enviando estímulos a la médula, desde la piel y el resto de órganos el hipotálamo es el termostato biológico. Las señales de frio se transmiten a través de las fibras delta A, el calor por las fibras C (no mielinizadas), para aumentar o disminuir la temperatura produciendo vasoconstricción y escalofrío en caso de hipotermia, y vasodilatación y sudoración en caso de hipertermia. (5)

\section{Las principales fuentes de perdida de calor son:}

- Evaporación, vaporización de agua a través de pérdidas insensibles y sudor.

- Radiación, emisión de energía electromagnética infrarroja.

- Conducción, transferencia directa de calor a un objeto frío adyacente.

- Convección, transferencia de calor directo a las corrientes de aire

- Mecanismos de intercambio de calor y relevancia en pacientes anestesiados

- Mecanismo Importancia, cuantitativa (\%) Radiación 65 Convección 25 Conducción 10

- Evaporación 10

\section{Generalidades}

La monitorización de la $\mathrm{T}^{\circ}$ corporal es imprescindible para facilitar el mantenimiento de la normotermia durante la cirugía y detectar a tiempo la aparición de hipotermia no intencionada. (6)

La principal causa de hipotermia en la mayoría de los pacientes es la redistribución del calor corporal desde el compartimiento central al periférico. La magnitud de esta transferencia de calor depende mayormente del gradiente térmico entre ambos compartimentos. (7)

Entre los factores más influyentes tenemos la edad, el peso, la duración de la cirugía y las temperaturas corporal y ambiental, por lo que los ancianos y los niños son los más susceptibles, los 
niños al tener una superficie corporal más amplia en relación a su perfil metabólico tienden a perder rápidamente calor.

Los obesos sufren menos pérdida de calor por que tienden a la vasodilatación lo que les hace generar más calor además de saber que la grasa tiene baja conductibilidad termal. (8)

La temperatura del quirófano tiene gran influencia ya que determina la velocidad a la que se pierde calor por radiación y convección desde la piel, y por evaporación desde el campo quirúrgico. Durante la primera hora de cirugía la temperatura desciende de1 a 1.6 grados centígrados, luego de esto se calcula que puede ocurrir un descenso de 1 grado por cada 2 horas de cirugía.

\section{Hipotermia y anestesia}

La inducción de la anestesia produce vasodilatación por la depresión de las respuestas vasoconstrictoras. El compartimiento central sufre una pérdida de calor progresiva, transfiriéndola al compartimiento periférico (redistribución). Estos cambios son directamente proporcional a la dosis de anestésicos endovenosos. (9)

La anestesia regional inhibe el control termorregulador de forma central, pero su acción periférica es mayor por el bloqueo de los nervios periféricos simpáticos y motores, inhibiendo la vasoconstricción termorreguladora y los escalofríos, La respuesta vasoconstrictora de los ancianos es de menor magnitud que la de los adultos jóvenes.

En la cirugía laparoscópica la perdida de calor durante la insuflación laparoscópica se debe principalmente a la evaporación del agua, con una pérdida de $0.3^{\circ} \mathrm{C}$ por cada 501 ts.

En la recesión transuretral de próstata la irrigación continua es un factorcoadyuvante en la instauración de hipotermia intraoperatoria sumado al hecho de la edad de los pacientes. 
Elio O. Ochoa-Gavilánez; Carmen S. Olave-Matamba; Jenny P. Guzmán-Arias; Pedro L. Maldonado-Álava

Durante la cirugía cardiaca y la circulación extracorpórea se inducen cambios en la temperatura tanto al inicio como al final de la misma por el enfriamiento y recalentamiento del compartimiento, la perdida de calor sobre todo del compartimento central y la difícil redistribución en el postoperatorio hace que sea difícil su manejo adecuado.

\section{Recomendaciones.}

La monitorización de la temperatura en el postoperatorio es deficiente, no hay registros de dicha constante vital. El objetivo en la preservación de calor consiste en minimizar las pérdidas de calor reduciendo la radiación y convección desde la piel

Recomiendan medir la temperatura una hora antes de la inducción, en el intraoperatorio cada 30min, en el postoperatorio al ingreso en reanimación y cada $15 \mathrm{~min}$ hasta que alcance los 36 .C, y luego cada hora hasta que alcance los 36,5 .C.

El precalentamiento es una recomendación actual, al impedir la pérdida de calor por redistribución.

Es más fácil mantener la normotermia intraoperatoria que recalentar a los pacientes en el postoperatorio ya que en este último La vasoconstricción periférica limita el flujo de calor hacia el compartimiento periférico.

Administración de fluidos precalentados se recomienda calentar las soluciones a 39 grados centígrados. 


\section{Bibliografía.}

1. Gutierrez S, Baptista W. Hipotermia postoperatoria inadvertida en la Sala de Recuperación Post Anestésica del Hospital de Clínicas "Dr. Manuel Quintela". Anestesia Analgesia Reanimación. 2006 2006; 21(1): p. 1 9 .

2. Reyes Perdomo JF. HIPOTERMIA Y ANESTESIA. In IX CONGRESO CENTROAMERICANO Y DEL CARIBRE DE ANESTESIOLOGIA; 2010; Guatemala. p. 1-13.

3. Hall JE. Guyton y Hall. Tratado de fisiología médica. 13th ed. Madrid: Elsevier; 2016.

4. Lauga A, Perrone SV. Monitoreo de las presiones de la arteria pulmonar. Insuficiencia cardíaca. 2007 sep; 2(3): p. 99-104.

5. De la Fuente RA, Quitero M. Sistema regulador del cuerpo humano. Ciencia y Desarrollo. 2009 nov; $39(2)$.

6. Biazzotto CB, Brudniewski M, Schmidt AP, Auler Junior JOC. Hipotermia no período peri-operatório. Revista Brasileira de Anestesiologia. 2006; 56(1): p. 86.

7. Baptista W, Rando K, Zunini G. Hipotermia perioperatoria. Anestesia Analgesia Reanimación. 2010 dic; 23(2).

8. Villegas González J, Villegas Arenas OA, Villegas González V. Semiología de los signos vitales: Una mirada novedosa a un problema vigente. Archivos de Medicina. 2012 dic; 12(2).

9. Fernández-Meré L, Álvarez-Blanco M. Manejo de la hipotermia perioperatoria. Rev Esp Anestesiol Reanim. 2012; 59(1): p. 379-389. 